\title{
Unsteady Couette Flow through a Porous Medium in a Rotating System
}

\author{
Maitree Jana ${ }^{1}$, Sanatan Das ${ }^{2}$, Rabindra Nath Jana ${ }^{1}$ \\ ${ }^{1}$ Department of Applied Mathematics, Vidyasagar University, Midnapore, India \\ ${ }^{2}$ Department of Mathematics, University of Gour Banga, English Bazar, India \\ Email: jana261171@yahoo.co.in
}

Received October 19, 2012; revised November 23, 2012; accepted December 5, 2012

\begin{abstract}
An investigation has been made on an unsteady Couette flow of a viscous incompressible fluid through a porous medium in a rotating system. The solution of the governing equations has been obtained by the use of Laplace transform technique. It is found that the primary velocity decreases and the magnitude of the secondary velocity increases with an increase in rotation parameter. The fluid velocity components are decelerated by an increase of Reynolds number. An increase in porosity parameter leads to increase the primary velocity and the magnitude of the secondary velocity. It is also found that the solution for small time converges more rapidly than the general solution. The asymptotic behavior of the solution is analyzed for small as well as large values of rotation parameter and Reynolds number. It is observed that a thin boundary layer is formed near the moving plate of the channel and the thicknesses of the boundary layer increases with an increase in porosity parameter.
\end{abstract}

Keywords: Couette Flow; Rotation Parameter; Reynolds Number; Porous Medium; Rotating System and Boundary Layer

\section{Introduction}

The flow between two parallel plates is a classical problem that has many applications in accelerators, aerodynamic heating, electrostatic precipitation, polymer technology, petroleum industry, purification of crude oil, fluid droplets and sprays. Such a flow model is of great interest, not only for its theoretical significance, but also for its wide applications to geophysics and engineering. A lot of research work concerning the flow between two parallel plates studied in a rotating system have appeared, for example, Batchelor [1], Ganapathy [3], Gupta [4] and Mazumder [5]. The flows through porous medium are very much prevalent in nature and therefore, the study of such flows has become of principal interest in many scientific and engineering applications. This type of flows has shown their great importance in petroleum engineering to study the movements of natural gas, oil and water through the oil reservoirs; in chemical engineering for the filtration and water purification processes. Further, to study the underground water resources and seepage of water in river beds one need the knowledge of the fluid flow through porous medium. Therefore, there are number of practical uses of the fluid flow through porous media. Rotation has an immense importance in various phenomena such as in cosmical fluid dynamics, meteor- ology, geophysical fluid dynamics, gaseous and nuclear reactors and many engineering applications, that is why, the study of Couette flow through porous medium in a rotating system enhances an interest to the researchers due to its applications in the aforesaid area. Such a study has a greater importance in the design of turbines and turbo mechanics, in estimating the flight path of rotating wheels and spin-stabilized missiles. A large number of investigations has been made on the flow through a porous medium in a rotating system. In general, most of solutions for unsteady flows of viscous fluids are in a series form. These series may be rapidly convergent for large values of the time but slowly convergent for small values of the time or vice versa. Sometimes, it can be difficult to obtain the solution for small values of the time but it can be easy to obtain it for large values of the time and the opposite can also be true. Vidyanidhi and Nigam [6] studied the channel flow between rotating parallel plates under constant pressure gradient. Jana and Dutta [7] studied the steady Couette flow of a viscous incompressible fluid between two infinite parallel plates, one stationary and the other moving with uniform velocity, in a rotating frame of reference. Singh and Sharma [8] have presented the three dimensional Couette flow through porous media. A periodic solution of oscillatory Couette flow through a porous medium in rotating sys- 
tem has been obtained by Singh et al. [9]. Guria et al. [10] have described the unsteady Couette flow in a rotating system. Das et al. [10] have studied the unsteady Couette flow with an oscillatory velocity of one of the plates in a rotating system. The unsteady MHD Couette flow in a rotating system has been investigated by Das et al. [12]. Attia [13] has studied the effect of porosity on unsteady Couette flow with heat transfer in the presence of uniform suction and injection. Israel-Cookey et al. [14] have presented the MHD oscillatory Couette flow of a radiating viscous fluid in a porous medium with periodic wall temperature. The unsteady hydromagnetic Couette flow through a porous medium in a rotating system have been presented Prasad and Kumar [15]. Das et al. [16] have studied the Couette flow through porous medium in a rotating system.

In the present paper, we have studied the unsteady Couette flow between two infinite horizontal parallel plates in a porous medium in a rotating system when one of the plate moving with uniform velocity and the other one held at rest. The fluid and plates are in a state of rigid body rotation with uniform angular velocity $\Omega$. The solutions for the velocity distributions as well as shear stresses have been obtained for small time as well as for large time by the Laplace transform technique. It is found that the primary velocity $u_{1}$ decreases and the magnitude of the secondary velocity $v_{1}$ increases with an increase in rotation parameter $K^{2}$. The primary velocity $u_{1}$ and the magnitude of the secondary velocity $v_{1}$ decrease with an increase in Reynolds number Re. An increase in porosity parameter $\sigma$ leads to increase in the values of both the primary velocity $u_{1}$ and the magnitude of the secondary velocity $v_{1}$. It is also found that the solution for small time converges more rapidly than the general solution. For the steady state solution, the asymptotic behavior of the solution is analysed for small as well as large values of rotation parameter $K^{2}$ and Reynolds number Re. It is observed that a thin boundary layer is formed near the stationary plate and the thicknesses of the boundary layer increases with an increase in porosity parameter $\sigma$.

\section{Mathematical Formulation and Its Solution}

Consider the unsteady flow of a viscous incompressible fluid between two infinite parallel porous plates embedded in a porous medium. The plates are separated by a distance $h$. The fluid and channel rotate in unison about an axis normal to the planes of the plates with a uniform angular velocity $\Omega$. Choose a Cartesian co-ordinate system with $x$-axis along the lower stationary plate in the direction of the flow, the $y$-axis is normal to the plates and the z-axis perpendicular to $x y$-plane (see Figure 1). Flow within the channel is induced due to the motion of the upper plate at $y=h$ parallel to itself in $x$-direction with a uniform velocity $u_{0}$. Initially, at time $t \leq 0$, the fluid as well as the plates of the channel are assumed to be at rest. At time $t>0$ the upper plate at $y=h$ starts moving with uniform velocity $u_{0}$ along $x$-direction in its own plane while the lower plate at $y=0$ is kept fixed. The velocity components are $(u, v, w)$ relative to a frame of reference rotating with the fluid. Since plates of the channel are infinitely long along $x$ and $y$ directions, all physical quantities will be functions of $z$ and $t$ only. The equation of continuity gives $\frac{\partial v}{\partial y}=0$ which on integration yields $v=$ constant $=-v_{0}$, where $v_{0}>0$ for suction and $v_{0}<0$ for the blowing at the plate.

The $x$-, $y$ - and $z$-components of Navier-Stokes equation are

$$
\begin{gathered}
\frac{\partial u}{\partial t}-v_{0} \frac{\partial u}{\partial y}-2 \Omega w=v \frac{\partial^{2} u}{\partial y^{2}}-\frac{v}{k^{\prime}} u, \\
0=-\frac{1}{\rho} \frac{\partial p}{\partial y}+\frac{v}{k^{\prime}} v_{0}, \\
\frac{\partial w}{\partial t}-v_{0} \frac{\partial w}{\partial y}+2 \Omega u=v \frac{\partial^{2} w}{\partial y^{2}}-\frac{v}{k^{\prime}} w,
\end{gathered}
$$

where $\rho$ and $v$ are respectively the fluid density, the kinematic viscosity and $k^{\prime}$ the permeability of the porous medium.

The initial and boundary conditions are

$$
\begin{aligned}
& u=w=0, v=-v_{0}, \text { for } 0 \leq y \leq h, t \leq 0, \\
& u=w=0, v=-v_{0}, \text { at } y=0 \text { for } t>0, \\
& u=u_{0}, w=0, v=-v_{0}, \text { at } y=h \text { for } t>0 .
\end{aligned}
$$

Introducing the non-dimensional variables

$$
\eta=\frac{y}{h}, u_{1}=\frac{u}{u_{0}}, w_{1}=\frac{v}{u_{0}}, \tau=\frac{v t}{h^{2}}
$$

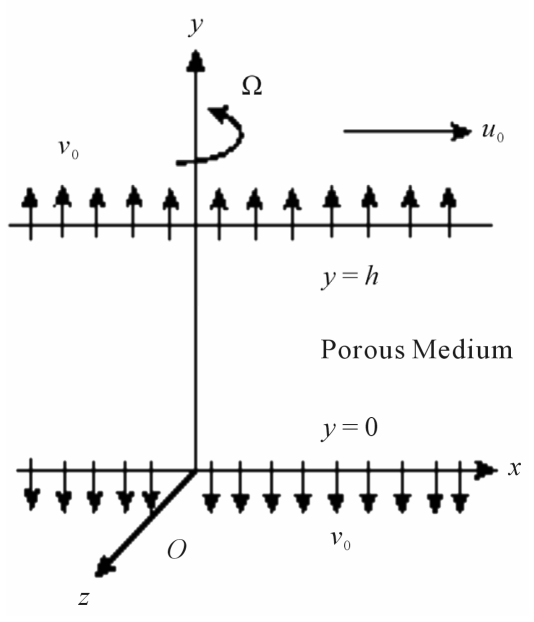

Figure 1. Geometry of the problem. 
Equations (1) and (3) become

$$
\begin{gathered}
\frac{\partial u_{1}}{\partial \tau}-\operatorname{Re} \frac{\partial u_{1}}{\partial \eta}-2 K^{2} w_{1}=\frac{\partial^{2} u_{1}}{\partial \eta^{2}}-\frac{1}{\sigma^{2}} u_{1}, \\
\frac{\partial w_{1}}{\partial \tau}-\operatorname{Re} \frac{\partial w_{1}}{\partial \eta}+2 K^{2} u_{1}=\frac{\partial^{2} w_{1}}{\partial \eta^{2}}-\frac{1}{\sigma^{2}} w_{1},
\end{gathered}
$$

where $K^{2}=\frac{\Omega h^{2}}{v}$ is the rotation parameter and $\operatorname{Re}=\frac{v_{0} h}{v}$ the Reynolds number and $\sigma^{2}=\frac{k^{\prime} u_{0}^{2}}{v^{2}}$ the porosity parameter.

Combing Equations (6) and (7), we have

$$
\frac{\partial q}{\partial \tau}-\operatorname{Re} \frac{\partial q}{\partial \eta}-2 \mathrm{i} K^{2} q=\frac{\partial^{2} q}{\partial \eta^{2}}-\frac{1}{\sigma^{2}} q
$$

where

$$
q=u_{1}+\mathrm{i} w_{1} \text { and } \mathrm{i}=\sqrt{-1} .
$$

The initial and boundary conditions for $q(\eta, \tau)$ are

$$
\begin{gathered}
q(\eta, 0)=0 \text { for } 0 \leq \eta \leq 1 \text { and } \tau \leq 0, \\
q(0, \tau)=0 \text { and } q(0, \tau)=1 \text { for } \tau>0 .
\end{gathered}
$$

Taking the Laplace transform, Equation (8) becomes

$$
\frac{\mathrm{d}^{2} \bar{q}}{\mathrm{~d} \eta^{2}}-\operatorname{Re} \frac{\mathrm{d} \bar{q}}{\mathrm{~d} \eta}+\left(s+2 \mathrm{i} K^{2}+\frac{1}{\sigma^{2}}\right) \bar{q}=0,
$$

where

$$
\bar{q}=\int_{0}^{\infty} q(\eta, \tau) \mathrm{e}^{-s \tau} d \tau .
$$

The boundary conditions for $\bar{q}(\eta, s)$ are

$$
\bar{q}(0, s)=0 \text { and } \bar{q}(1, s)=\frac{1}{s} .
$$

The solution of Equation (12) subject to the boundary conditions (14) is

$$
\bar{q}(\eta, s)=\frac{1}{s} \frac{\sinh \sqrt{s+a} \eta}{\sinh \sqrt{s+a}} \mathrm{e}^{\frac{1}{2} \operatorname{Re}(1-\eta)},
$$

where

$$
a=\frac{\mathrm{Re}^{2}}{4}+\frac{1}{\sigma^{2}}+2 \mathrm{i} K^{2} .
$$

The inverse Laplace's transform of Equation (15) is

$$
q(\eta, \tau)=\left[\frac{\sinh (\alpha+\mathrm{i} \beta) \eta}{\sinh (\alpha+\mathrm{i} \beta)}+\sum_{n=1}^{\infty} \frac{2 n \pi(-1)^{n} \mathrm{e}^{-\lambda_{n}^{2} \tau}}{n^{2} \pi^{2}+(\alpha+\mathrm{i} \beta)^{2}} \sin n \pi \eta\right] \mathrm{e}^{\frac{1}{2} \operatorname{Re}(1-\eta)}
$$

where

$$
\lambda_{n}^{2}=n^{2} \pi^{2}+(\alpha+\mathrm{i} \beta)^{2}, \quad \alpha, \beta=\frac{1}{\sqrt{2}}\left[\left\{\left(\frac{\mathrm{Re}^{2}}{4}+\frac{1}{\sigma^{2}}\right)^{2}+4 K^{4}\right\}^{\frac{1}{2}} \pm\left(\frac{\mathrm{Re}^{2}}{4}+\frac{1}{\sigma^{2}}\right)\right]^{\frac{1}{2}} .
$$

On separating into a real and imaginary parts, we get

$$
\begin{aligned}
u_{1}= & \mathrm{e}^{\frac{1}{2} \operatorname{Re}(1-\eta)}\left[\frac{S(\alpha \eta) S(\alpha)+C(\alpha \eta) C(\alpha)}{S^{2}(\alpha)+C^{2}(\alpha)}+2 \sum_{n=1}^{\infty} \frac{n \pi(-1)^{n} \sin n \pi \eta}{\left(n^{2} \pi^{2}+\alpha^{2}-\beta^{2}\right)^{2}+4 \alpha^{2} \beta^{2}}\right. \\
& \left.\times\left\{\left(n^{2} \pi^{2}+\alpha^{2}-\beta^{2}\right) \cos 2 \alpha \beta \tau-2 \alpha \beta \sin 2 \alpha \beta \tau\right\} \times \mathrm{e}^{-\left(n^{2} \pi^{2}+\alpha^{2}-\beta^{2}\right) \tau}\right], \\
w_{1}= & \mathrm{e}^{\frac{1}{2} \operatorname{Re}(1-\eta)}\left[\frac{C(\alpha \eta) S(\alpha)-S(\alpha \eta) C(\alpha)}{S^{2}(\alpha)+C^{2}(\alpha)}-2 \sum_{n=1}^{\infty} \frac{n \pi(-1)^{n} \sin n \pi \eta}{\left(n^{2} \pi^{2}+\alpha^{2}-\beta^{2}\right)^{2}+4 \alpha^{2} \beta^{2}}\right. \\
& \left.\times\left\{2 \alpha \beta \cos 2 \alpha \beta \tau+\left(n^{2} \pi^{2}+\alpha^{2}-\beta^{2}\right) \sin 2 \alpha \beta \tau\right\} \times \mathrm{e}^{-\left(n^{2} \pi^{2}+\alpha^{2}-\beta^{2}\right) \tau}\right],
\end{aligned}
$$

where

$$
\begin{aligned}
& S(\alpha \eta)=\sinh \alpha \eta \cos \beta \eta, C(\alpha \eta)=\cosh \alpha \eta \sin \beta \eta, \\
& S(\alpha)=\sinh \alpha \cos \beta, C(\alpha)=\cosh \alpha \sin \beta .
\end{aligned}
$$

The solution given by Equations (19) and (20) exists for both $\mathrm{Re}<0$ (corresponding to $v_{0}<0$ for the blowing at the plates) and $\operatorname{Re}>0$ (corresponding to $v_{0}>0$ for the suction at the plates).

\section{Solutions for Small Time}

Following Carslaw and Jaegar [17], for small time, the solution of (12) subject to the boundary conditions (14) is obtained by Laplace transform technique in the following form 


$$
\begin{aligned}
& q(\eta, \tau)=\mathrm{e}^{-\left(\frac{1}{\sigma^{2}}+2 \mathrm{i} K^{2}\right) \tau} \mathrm{e}^{\frac{1}{2} \operatorname{Re}(1-\eta)} \sum_{k=0}^{\infty} \sum_{n=0}^{\infty}\left(\frac{1}{\sigma^{2}}+2 \mathrm{i} K^{2}\right)^{n}(4 \tau)^{n} \\
& \times\left[\left\{j^{2 n} \operatorname{erfc}\left(\frac{c}{2 \sqrt{\tau}}\right)-j^{2 n} \operatorname{erfc}\left(\frac{d}{2 \sqrt{\tau}}\right)\right\}\right. \\
& \left.-\frac{\operatorname{Re}^{2}}{8} \sqrt{\tau}\left\{c j^{2 n+1} \operatorname{erfc}\left(\frac{c}{2 \sqrt{\tau}}\right)-d j^{2 n+1} \operatorname{erfc}\left(\frac{d}{2 \sqrt{\tau}}\right)\right\}\right]
\end{aligned}
$$

where

$$
\begin{gathered}
c=2 m+1-\eta, d=2 m+1+\eta, \\
j^{n} \operatorname{erfc}(x)=\int_{x}^{\infty} j^{n-1} \operatorname{erfc}(\xi) d \xi
\end{gathered}
$$

$$
j \operatorname{erfc}(x)=\int_{x}^{\infty} \operatorname{erfc}(\xi) \mathrm{d} \xi, j^{0} \operatorname{erfc}(x)=\operatorname{erfc}(x) .
$$

The solution (22) can be written as

$$
\begin{aligned}
& q(\eta, \tau)=\mathrm{e}^{-\left(\frac{1}{\sigma^{2}}+2 \mathrm{i} K^{2}\right) \tau} \mathrm{e}^{\frac{1}{2} \operatorname{Re}(1-\eta)} \sum_{k=0}^{\infty}\left(\frac{1}{\sigma^{2}}+2 \mathrm{i} K^{2}\right)^{n}(4 \tau)^{n} T_{r}, \\
& r=0,2,4,6, \cdots,
\end{aligned}
$$

where

$$
\begin{aligned}
T_{r}= & \sum_{k=0}^{\infty}\left[\left\{j^{r} \operatorname{erfc}\left(\frac{c}{2 \sqrt{\tau}}\right)-j^{r} \operatorname{erfc}\left(\frac{d}{2 \sqrt{\tau}}\right)\right\}\right. \\
& \left.-\frac{\operatorname{Re}^{2}}{4} \sqrt{\tau}\left\{c j^{r+1} \operatorname{erfc}\left(\frac{c}{2 \sqrt{\tau}}\right)-d j^{r+1} \operatorname{erfc}\left(\frac{d}{2 \sqrt{\tau}}\right)\right\}\right],
\end{aligned}
$$

$r=0,2,4,6, \cdots$

On separating into a real and imaginary parts, we get the velocity distributions for the primary and the secondary flow as

$$
u_{1}=\mathrm{e}^{\frac{1}{2} \operatorname{Re}(1-\eta)}\left[P(\eta, \tau) \cos 2 K^{2} \tau+Q(\eta, \tau) \sin 2 K^{2} \tau\right] \mathrm{e}^{-\frac{\tau}{\sigma^{2}}},
$$

$$
w_{1}=\mathrm{e}^{\frac{1}{2} \operatorname{Re}(1-\eta)}\left[Q(\eta, \tau) \cos 2 K^{2} \tau-P(\eta, \tau) \sin 2 K^{2} \tau\right] \mathrm{e}^{-\frac{\tau}{\sigma^{2}}},
$$

where

$$
\begin{aligned}
P(\eta, \tau)= & T_{0}+\frac{1}{\sigma^{2}}(4 \tau) T_{2}+\left(\frac{1}{\sigma^{4}}-4 K^{4}\right)(4 \tau)^{2} T_{4} \\
& +\left(\frac{1}{\sigma^{6}}-\frac{12 K^{4}}{\sigma^{2}}\right)(4 \tau)^{3} T_{6}+\cdots \\
Q(\eta, \tau)= & 2 K^{2}(4 \tau) T_{2}+\frac{4 K^{2}}{\sigma^{2}}(4 \tau)^{2} T_{4} \\
& +\left(\frac{6 K^{2}}{\sigma^{4}}-8 K^{6}\right)(4 \tau)^{3} T_{6}+\cdots
\end{aligned}
$$

Equations (25) and (26) describe the fluid velocities for small time.

\section{Results and Discussion}

To study the effects of rotation, Reynolds number and porosity parameter on the velocity distributions we have presented the non-dimensional velocity components $u_{1}$ and $w_{1}$ against $\eta$ in Figures 2-6 for several values of the rotation parameter $K^{2}$, Reynolds number Re, porosity parameter $\sigma$ and time $\tau$. It is seen from Figure 2 that the primary velocity $u_{1}$ decreases and the magnitude of the secondary velocity $w_{1}$ increases with an increase in rotation number $K^{2}$. Figure 3 reveals that both the primary velocity $u_{1}$ and the magnitude of the secondary velocity $w_{1}$ decreases with increase in Reynolds number Re. It is observed from Figure 4 that both the primary velocity $u_{1}$ and the magnitude of the secondary velocity $w_{1}$ increase with an increase in porosity parameter $\sigma$. The presence of porous medium produces a resisting force in the flow field. So, the resistance in the

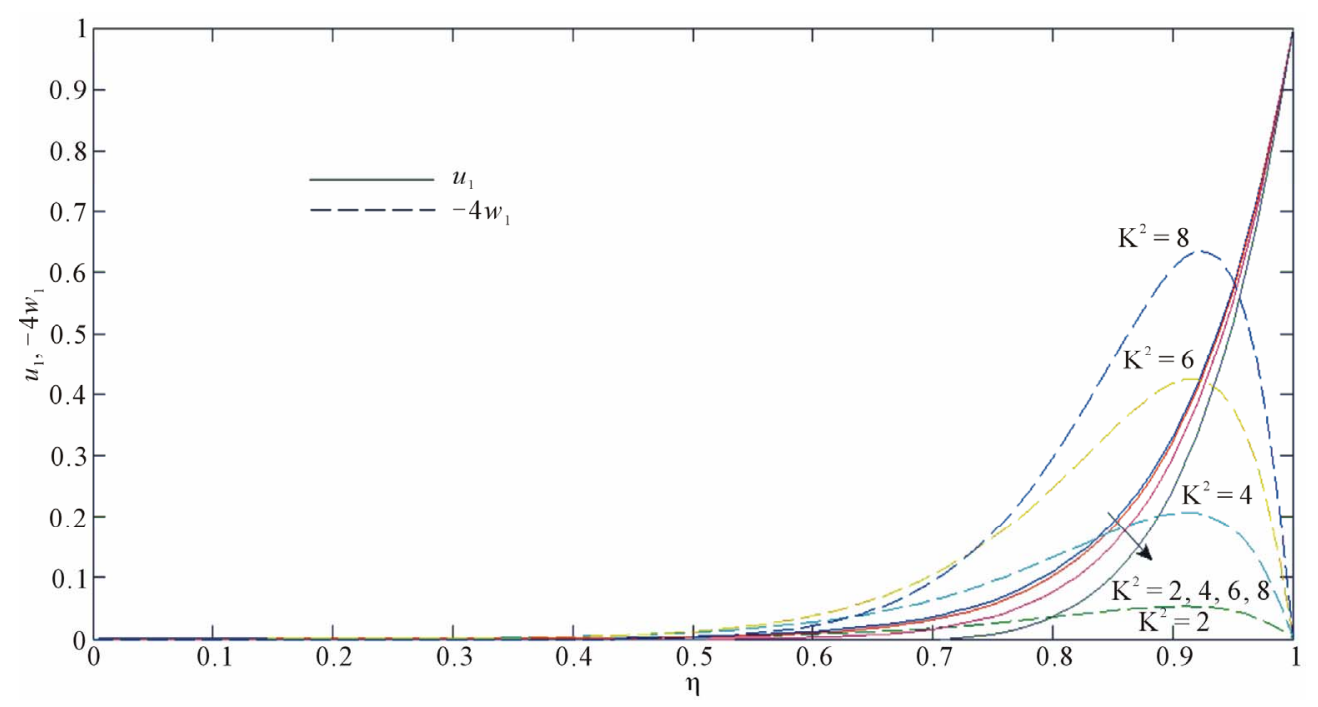

Figure 2. Velocities $u_{1}$ and $w_{1}$ for different $K^{2}$ when $\operatorname{Re}=2, \sigma=0.1$ and $\tau=0.2$. 


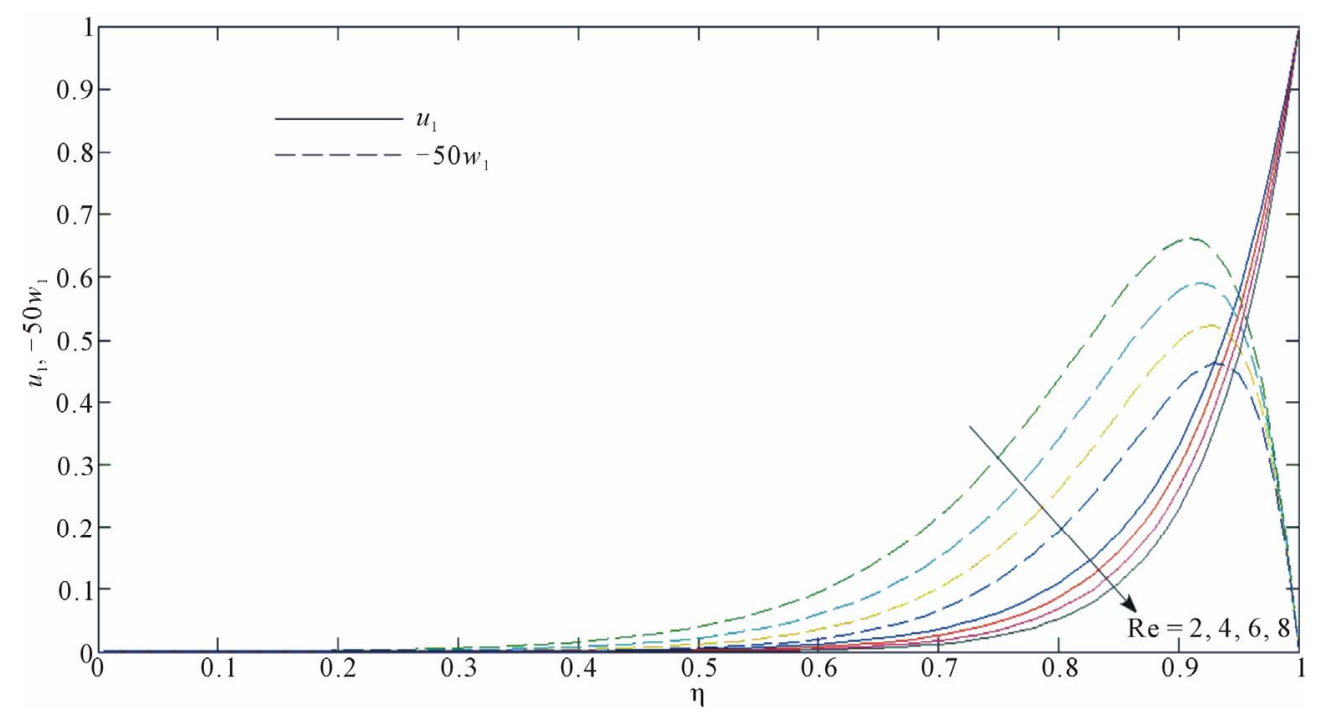

Figure 3. Velocities $u_{1}$ and $w_{1}$ for different Re only when $K^{2}=2, \sigma=0.1$ and $\tau=0.2$.

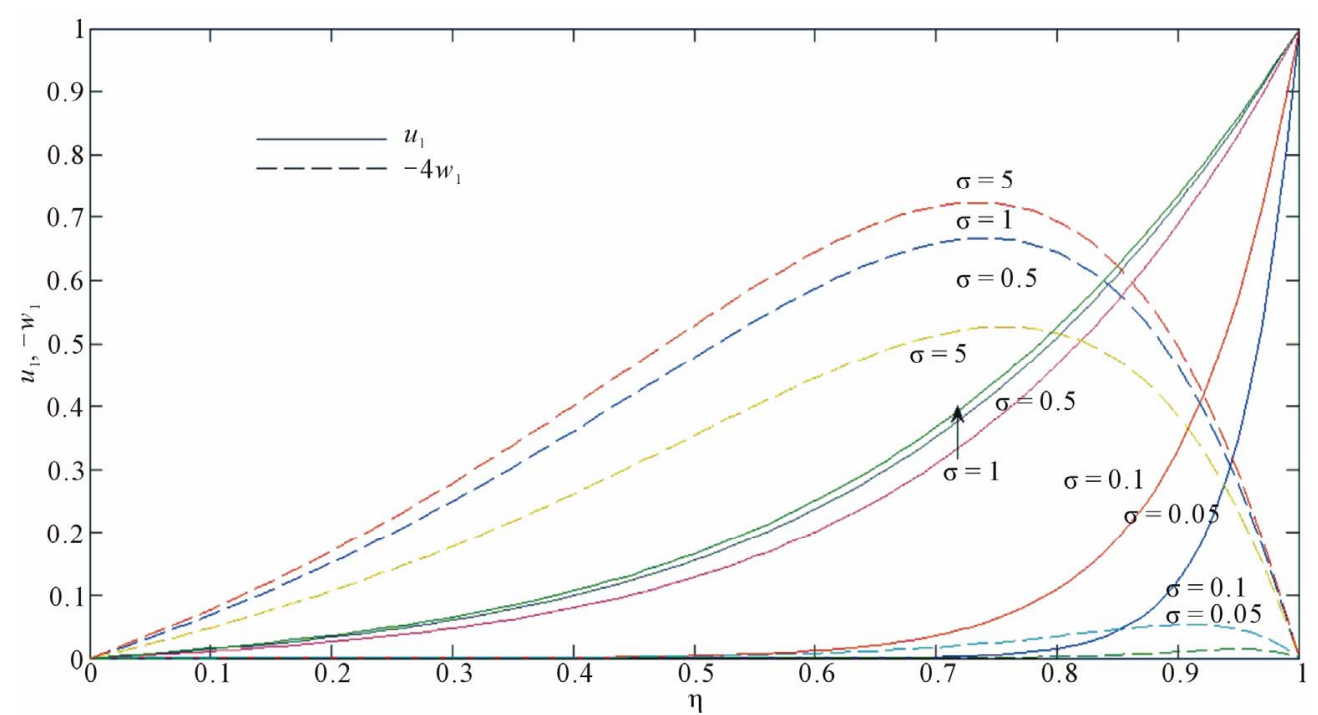

Figure 4. Velocities $u_{1}$ and $w_{1}$ for different $\sigma$ when $K^{2}=2, \operatorname{Re}=2$ and $\tau=0.2$.

flow field decreases as the porosity parameter $\sigma$ increases. This indicates that porosity of the medium has an accelerating influence on the flow field. Thus we can control the velocity field by introducing porous medium in a rotating system. It is observed from Figure 5 that both the primary velocity $u_{1}$ and the magnitude of the secondary velocity $w_{1}$ increases with an increase in times $\tau$. For small values of time, we have drawn the velocity components $u_{1}$ and $w_{1}$ on using the solution given by Equations (25) and (26) and the general solution given by Equations (19) and (20) in Figures 6 and 7. It is seen that the solution for small time given by Equations (25) and (26) converges more rapidly than the general solution given by (19) and (20). Hence, we conclude that for small times, the numerical values of the velocity components can be evaluated from Equations (25) and
(26) instead of Equations (19) and (20).

The non-dimensional shear stresses at the stationary plate $(\eta=0)$ due to the primary and the secondary flows are given by

$$
\begin{aligned}
& \tau_{x}+\mathrm{i} \tau_{y}=\left(\frac{\partial q}{\partial \eta}\right)_{\eta=0} \\
& =\mathrm{e}^{-\frac{1}{2} R e}\left[\frac{(\alpha+\mathrm{i} \beta)}{\sinh (\alpha+\mathrm{i} \beta)}+\sum_{n=1}^{\infty} \frac{2 n^{2} \pi^{2}(-1)^{n} \mathrm{e}^{-\left\{n^{2} \pi^{2}+(\alpha+\mathrm{i} \beta)^{2}\right\} \tau}}{n^{2} \pi^{2}+(\alpha+\mathrm{i} \beta)^{2}}\right]
\end{aligned}
$$

On separating into a real and imaginary parts, we get the shear stress components due to the primary and secondary flows at the stationary plate $\eta=0$ as 


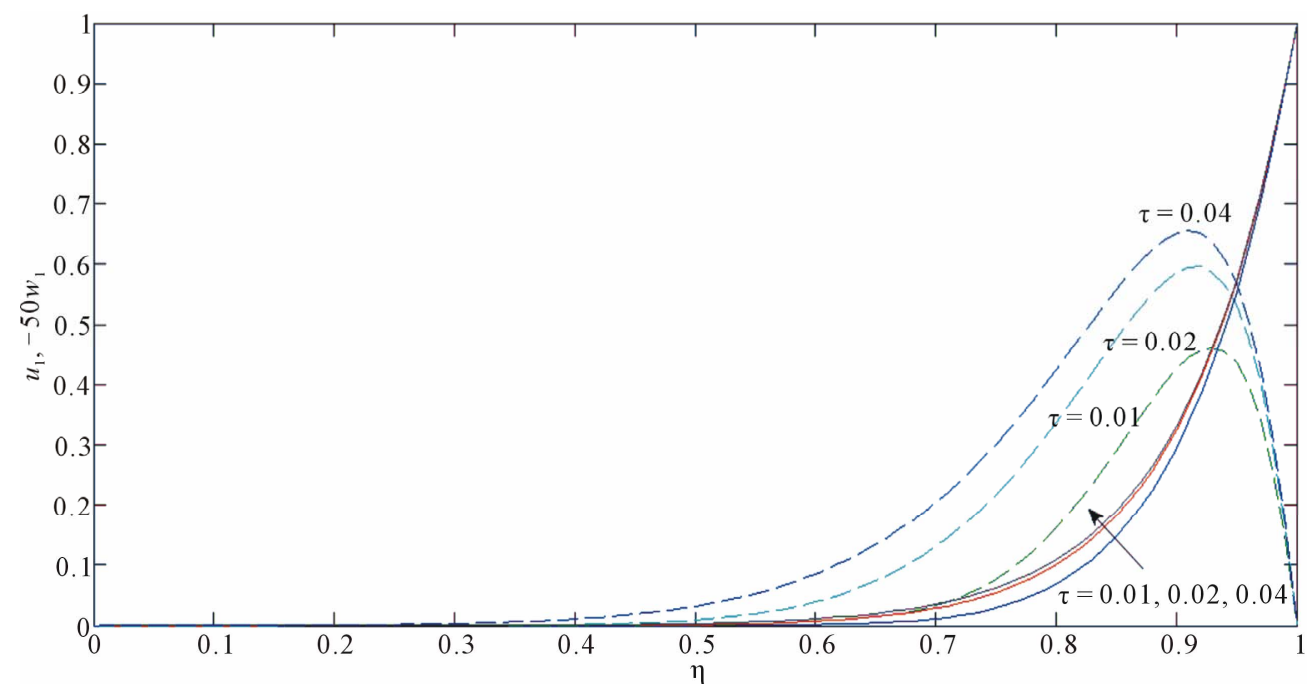

Figure 5. Velocities $u_{1}$ and $w_{1}$ for different time $\tau$ when $K^{2}=2, \operatorname{Re}=2$ and $\sigma=0.1$.

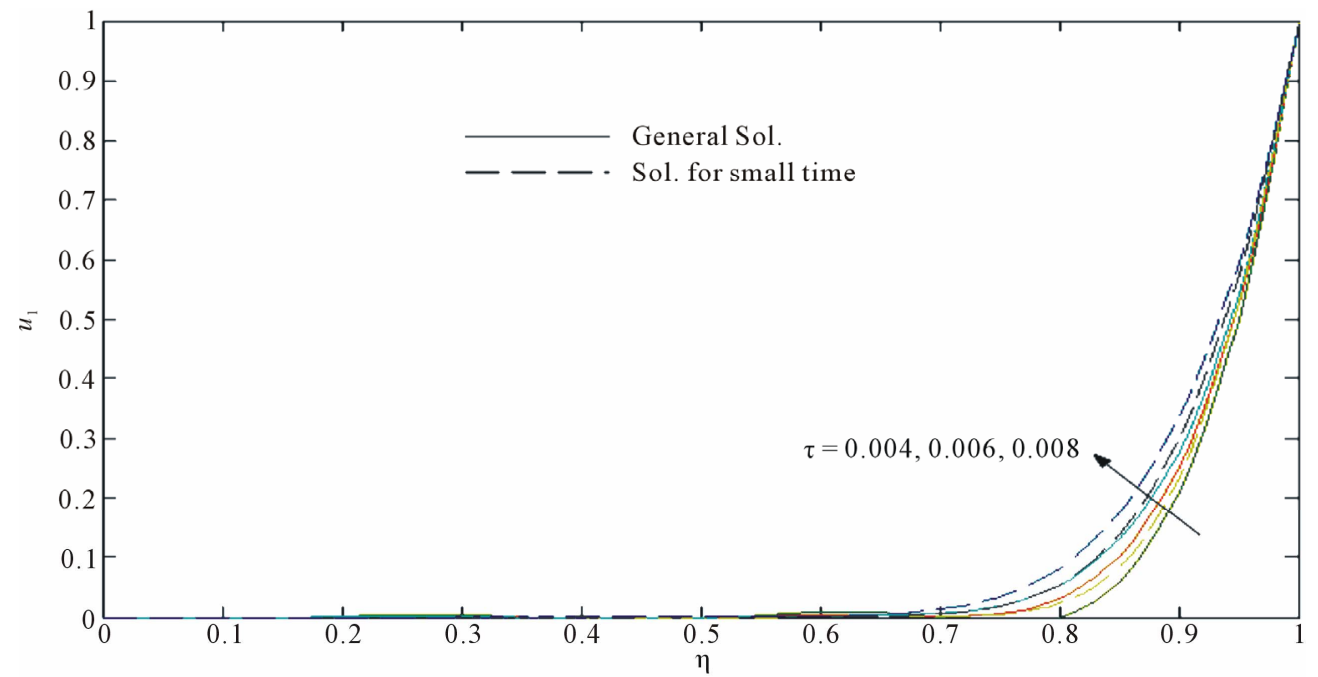

Figure 6. Velocity $u_{1}$ for general solution and solution for small time when $K^{2}=2, \operatorname{Re}=2$ and $\sigma=0.1$.

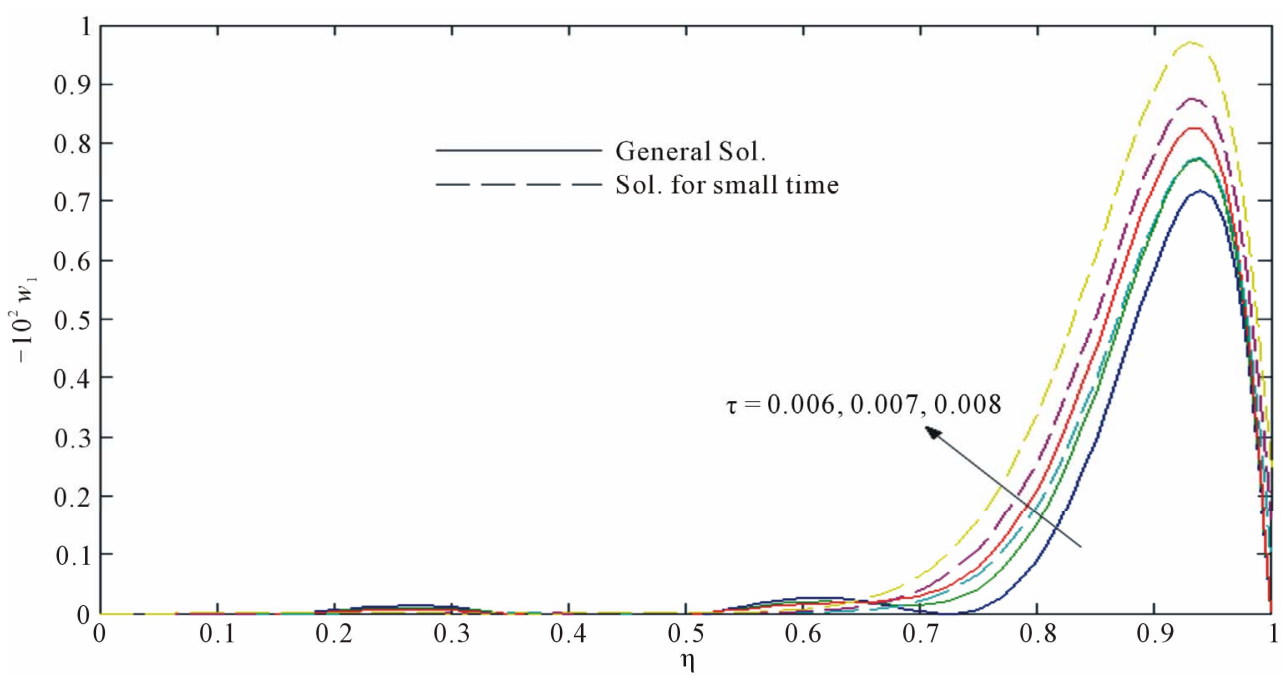

Figure 7. Velocity $w_{1}$ for general solution and solution for small time when $K^{2}=2, \operatorname{Re}=2$ and $\sigma=0.1$. 


$$
\begin{aligned}
\tau_{x}= & \mathrm{e}^{\frac{1}{2} \operatorname{Re}}\left[\frac{2(\alpha \sinh \alpha \cos \beta+\beta \cosh \alpha \sin \beta)}{\cosh 2 \alpha-\cos 2 \beta}+\sum_{n=1}^{\infty} \frac{2 n^{2} \pi^{2}(-1)^{n}}{\left(n^{2} \pi^{2}+\alpha^{2}-\beta^{2}\right)^{2}+4 \alpha^{2} \beta^{2}}\right. \\
& \left.\times\left\{\left(n^{2} \pi^{2}+\alpha^{2}-\beta^{2}\right) \cos 2 \alpha \beta \tau-2 \alpha \beta \sin 2 \alpha \beta \tau\right\} \times \mathrm{e}^{-\left(n^{2} \pi^{2}+\alpha^{2}-\beta^{2}\right) \tau}\right] \\
\tau_{y}= & \mathrm{e}^{\frac{1}{2} \operatorname{Re}}\left[\frac{2(\beta \sinh \alpha \cos \beta-\alpha \cosh \alpha \sin \beta)}{\cosh 2 \alpha-\cos 2 \beta}-\sum_{n=1}^{\infty} \frac{2 n^{2} \pi^{2}(-1)^{n}}{\left(n^{2} \pi^{2}+\alpha^{2}-\beta^{2}\right)^{2}+4 \alpha^{2} \beta^{2}}\right. \\
& \left.\times\left\{2 \alpha \beta \cos 2 \alpha \beta \tau+\left(n^{2} \pi^{2}+\alpha^{2}-\beta^{2}\right) \sin 2 \alpha \beta \tau\right\} \times \mathrm{e}^{-\left(n^{2} \pi^{2}+\alpha^{2}-\beta^{2}\right) \tau}\right]
\end{aligned}
$$

The numerical values of the non-dimensional shear stresses $\tau_{x}$ and $\tau_{y}$ at the plate $\eta=0$ are presented in Figures 8-10 against Reynolds number Re for several values of $K^{2}, \sigma$ and $\tau$. It is seen from Figure 8 that both the absolute value of the shear stresses $\tau_{x}$ and $\tau_{y}$ increase with increase in rotation parameter $K^{2}$ while the absolute value of the shear stresses $\tau_{x}$ increases and that of the shear stress $\tau_{y}$ decreases with an increase in Re. Reynolds number ( $\mathrm{Re})$ is the ratio of inertial forces to viscous forces and quantifies the relative importance of these two types of forces for given flow conditions. The viscous forces are dominant at low Reynolds numbers while the inertial forces are dominant at high Reynolds numbers. It is a good agreement with the physical point of view. Figures 9 and $\mathbf{1 0}$ show that the absolute value of the shear stress $\tau_{x}$ increases while the absolute value of the shear stress $\tau_{y}$ increases with an increase in either porosity parameter $\sigma$ or time $\tau$.

For small times, the non-dimensional shear stresses due to the primary and secondary flows at the stationary plate $(\eta=0)$ are given by

$$
\begin{aligned}
& \tau_{x}+\mathrm{i} \tau_{y}=\left(\frac{\partial q}{\partial \eta}\right)_{\eta=0} \\
& =\mathrm{e}^{-\left(\frac{1}{\sigma^{2}}+2 \mathrm{i} K^{2}\right) \tau} \sum_{n=0}^{\infty}\left(\frac{1}{\sigma^{2}}+2 \mathrm{i} K^{2}\right)^{n}(4 \tau)^{n} Y_{2 r-1}, \\
& r=0,1,2,3, \cdots
\end{aligned}
$$

where

$$
\begin{aligned}
Y_{2 r-1}= & \sum_{m=0}^{\infty}\left[\frac{1}{\sqrt{\tau}} j^{2 r-1} \operatorname{erfc}\left(\frac{2 m+1}{2 \sqrt{\tau}}\right)\right. \\
& -\frac{1}{8}(2 m+1) \operatorname{Re}^{2} j^{2 r} \operatorname{erfc}\left(\frac{2 m+1}{2 \sqrt{\tau}}\right) \\
& \left.+\frac{\operatorname{Re}^{2} \sqrt{\tau}}{4} j^{2 r+1} \operatorname{erfc}\left(\frac{2 m+1}{2 \sqrt{\tau}}\right)\right] .
\end{aligned}
$$

On separating into a real and imaginary parts, we get the shear stress components due to the primary and secondary flows as

$$
\begin{aligned}
& \tau_{x}=\mathrm{e}^{\frac{1}{2} \operatorname{Re}}\left[C(0, \tau) \cos 2 K^{2} \tau+D(0, \tau) \sin 2 K^{2} \tau\right] \mathrm{e}^{-\frac{\tau}{\sigma^{2}}}, \\
& \tau_{y}=\mathrm{e}^{\frac{1}{2} \operatorname{Re}}\left[D(0, \tau) \cos 2 K^{2} \tau-C(0, \tau) \sin 2 K^{2} \tau\right] \mathrm{e}^{-\frac{\tau}{\sigma^{2}}},
\end{aligned}
$$

where

$$
\begin{aligned}
C(0, \tau)= & Y_{-1}+\frac{1}{\sigma^{2}}(4 \tau) Y_{1}+\left(\frac{1}{\sigma^{4}}-4 K^{4}\right)(4 \tau)^{2} Y_{3} \\
& +\left(\frac{1}{\sigma^{6}}-\frac{12 K^{4}}{\sigma^{2}}\right)(4 \tau)^{3} Y_{5}+\cdots \\
D(0, \tau)= & 2 K^{2}(4 \tau) Y_{1}+\frac{4 K^{2}}{\sigma^{2}}(4 \tau)^{2} Y_{3} \\
& +\left(\frac{6 K^{2}}{\sigma^{4}}-8 K^{6}\right)(4 \tau)^{3} Y_{5}+\cdots
\end{aligned}
$$

For small time, the numerical values of the shear stress components calculated from Equations (29), (30), (33) and (34) are given in Tables $\mathbf{1}$ and $\mathbf{2}$ for several values of $\operatorname{Re}$ and $\tau$. It is observed that for small time the shear stresses calculated from Equations (33) and (34) give better result than that calculated from Equations (29) and (30). Hence, we conclude for small times shear stress components should be evaluated from Equations (33) and (34) instead of Equations (29) and (30).

We shall now discuss the asymptotic behavior of the solutions (25) and (26) for small and large values of $K^{2}$ and $\mathrm{Re}$, when the motion is in steady state. In the steady state $(\tau \rightarrow \infty)$, Equation (17) becomes

$$
q(\eta, \tau)=\frac{\sinh (\alpha+\mathrm{i} \beta) \eta}{\sinh (\alpha+\mathrm{i} \beta)} .
$$

Case 1): When $K^{2} \ll 1$ and $\operatorname{Re} \gg 1$.

When Re is large and $K^{2}$ is of small order of magnitude, the flow becomes boundary layer type. For the boundary layer flow near the upper plate $\eta=1$, introducing the boundary layer coordinate $\xi=1-\eta$, we obtain the velocity distributions from (36) as

$$
u_{1}=\mathrm{e}^{-\left(\frac{\mathrm{Re}}{2}+\alpha\right) \xi} \cos \beta \xi,
$$




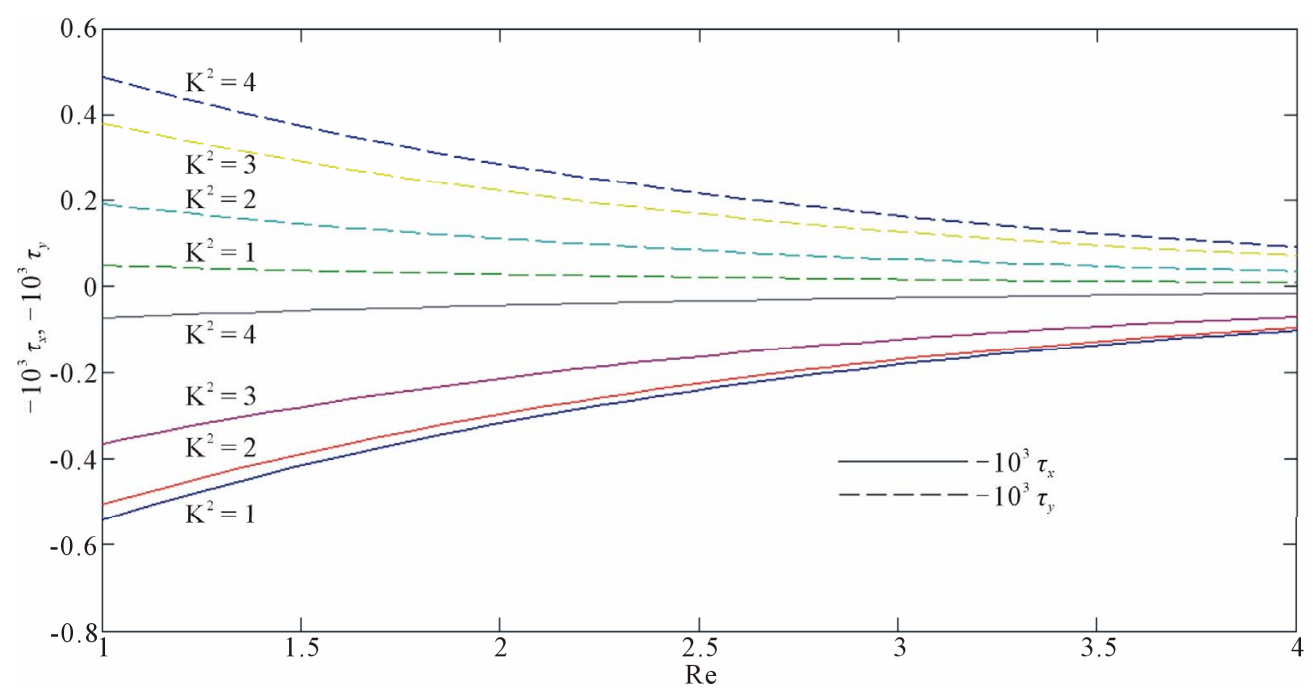

Figure 8. Shear stresses $\tau_{x}$ and $\tau_{y}$ for different $K^{2}$ when $\sigma=0.1$ and $\tau=0.2$.

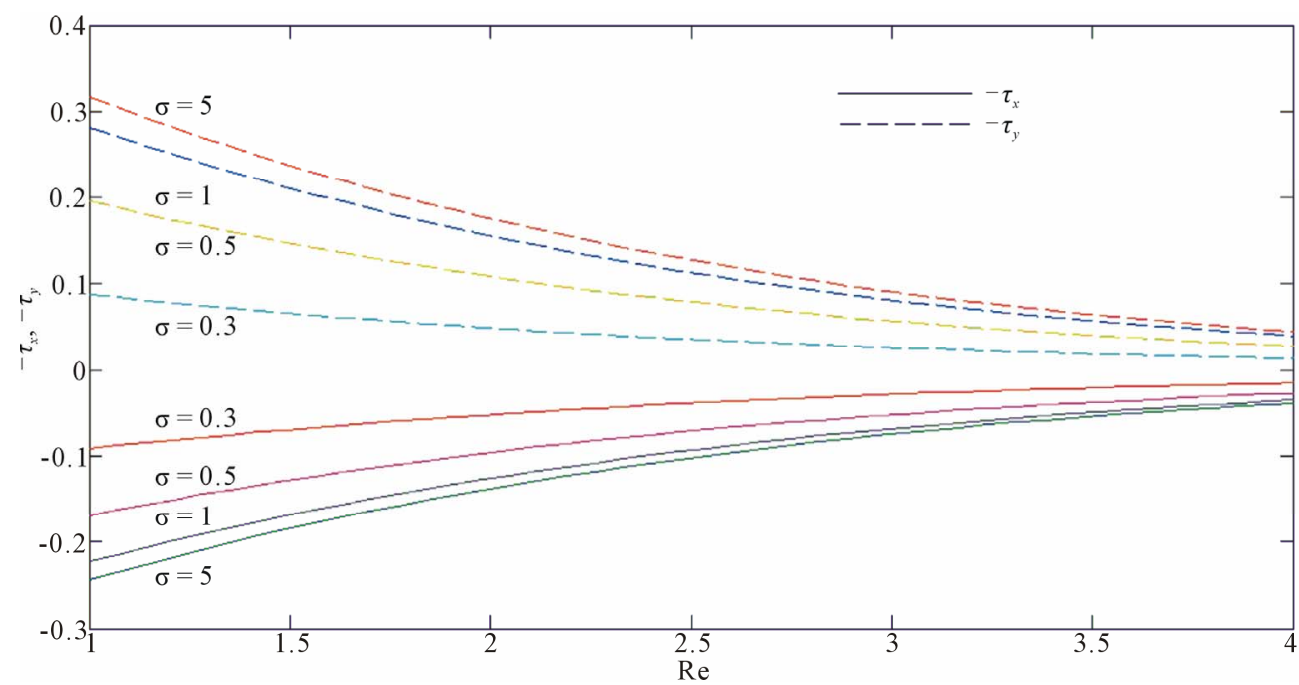

Figure 9. Shear stresses $\tau_{x}$ and $\tau_{y}$ for different $\sigma$ when $K^{2}=2$ and $\tau=0.2$.

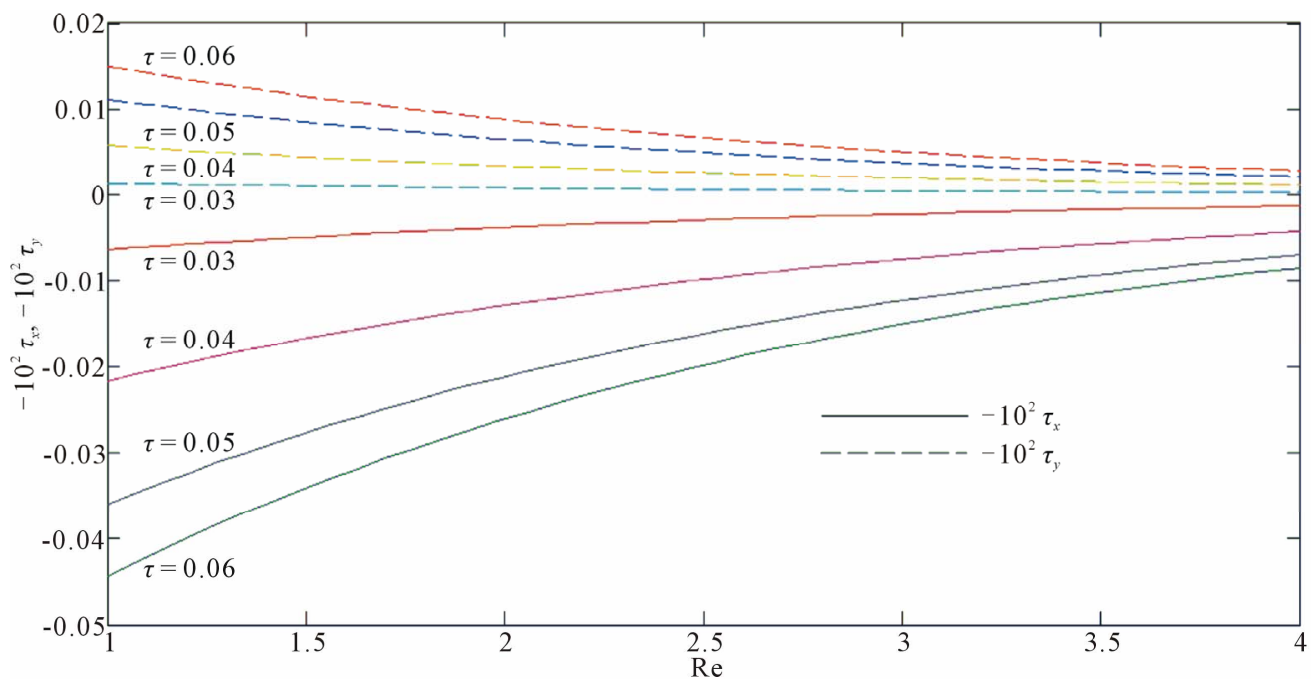

Figure 10. Shear stresses $\tau_{x}$ and $\tau_{y}$ for different $\tau$ when $K^{2}=2$ and $\sigma=0.1$. 
Table 1. Shear stress $10 \tau_{x}$ due to primary flow when $K^{2}=2$ and $\sigma=0.1$.

\begin{tabular}{ccccccc}
\hline \multirow{2}{*}{$\operatorname{Re} \backslash \tau$} & \multicolumn{3}{c}{ General solution } & \multicolumn{3}{c}{ Solution for small times } \\
\cline { 2 - 7 } & 0.04 & 0.05 & 0.06 & 0.04 & 0.05 & 0.06 \\
\hline 1 & 0.00217 & 0.00359 & 0.00443 & 0.03107 & 0.02736 & 0.01407 \\
2 & 0.00129 & 0.00212 & 0.00261 & 0.10398 & 0.09122 & 0.04630 \\
3 & 0.00075 & 0.00123 & 0.00150 & 0.26266 & 0.22920 & 0.11421 \\
4 & 0.00043 & 0.00070 & 0.00085 & 0.59325 & 0.51417 & 0.25021 \\
\hline
\end{tabular}

Table 2. Shear stress $-10^{2} \tau_{y}$ due to secondary flow when $K^{2}=2$ and $\sigma=0.1$.

\begin{tabular}{cccccccc}
\hline & \multicolumn{3}{c}{ General solution } & \multicolumn{3}{c}{ Solution for small times } \\
\cline { 2 - 7 } $\operatorname{Re}$ & 0.04 & 0.05 & 0.06 & 0.04 & 0.05 & 0.06 \\
\hline 1 & 0.00579 & 0.01111 & 0.01502 & 0.01037 & 0.02393 & 0.02489 \\
2 & 0.00342 & 0.00654 & 0.00882 & 0.03423 & 0.07917 & 0.07952 \\
4 & 0.00199 & 0.00378 & 0.00507 & 0.08480 & 0.19675 & 0.19852 \\
\hline
\end{tabular}

$$
w_{1}=-\mathrm{e}^{-\left(\frac{\mathrm{Re}}{2}+\alpha\right) \xi} \sin \beta \xi,
$$

where

$$
\alpha=\frac{\operatorname{Re}}{2}\left(1+\frac{4}{\operatorname{Re}^{2} \sigma^{2}}\right), \beta=\frac{4 K^{2}}{\operatorname{Re}} .
$$

It is evident from Equations (37) and (38) that there exists a single-deck boundary layer of thickness of order $O\left\{\left(\frac{\operatorname{Re}}{2}+\alpha\right)^{-1}\right\}$ near the moving plate of the channel where $\alpha$ is given by (39). The thickness of this boundary layer decreases with an increase in porosity parameter $\sigma$ since $\alpha$ decreases with increase in $\sigma$.

Case 2): When $K^{2} \gg 1$ and $R e \ll 1$.

In this case, the velocity distributions are obtained from the Equations (36) as

$$
\begin{aligned}
& u_{1}=\mathrm{e}^{-\left(\frac{\mathrm{Re}}{2}+\alpha\right) \xi} \cos \beta \xi, \\
& w_{1}=-\mathrm{e}^{-\left(\frac{\mathrm{Re}}{2}+\alpha\right) \xi} \sin \beta \xi,
\end{aligned}
$$

where

$$
\alpha, \beta=K\left[1 \pm \frac{1}{4 K^{2}}\left(\frac{\mathrm{Re}^{2}}{4}+\frac{1}{\sigma^{2}}\right)\right]
$$

Equations (40) and (41) show that there exists a singledecker boundary layer of thickness of order

$O\left\{\left(\frac{\operatorname{Re}}{2}+\alpha\right)^{-1}\right\}$ adjacent to the moving plate of the channel where $\alpha$ is given by (42). The thicknesses of the layer decreases with an increase in Reynolds number $\mathrm{Re}$ while it increases with increase in porosity parameter $\sigma$.

\section{Conclusion}

The unsteady Couette flow of a viscous incompressible fluid through a porous medium in a rotating system has been investigated. It is found that the primary velocity decreases and the magnitude of the secondary velocity increases with an increase in rotation parameter. The fluid velocity components decrease with an increase in Reynolds number. An increase in the porosity of the medium both the primary and the secondary velocities increase. That is, the porosity of the medium has an accelerating influence on the flow field. In turn, it can control the velocity field by introducing porous medium in a rotating system. It is also found that the solution for small time converges more rapidly than the general solution. For steady state, the asymptotic behavior of the solution is analyzed for small as well as large values of rotation parameter and Reynolds number. It is observed that a thin boundary layer is formed near the moving plate of the channel and the thicknesses of the layer increases with an increase in porosity parameter.

\section{REFERENCES}

[1] G. K. Batchelor, "An Introduction to Fluid Dynamics," Cambridge University Press, Cambridge, 1967.

[2] G. S. Seth, R. N. Jana and M. K. Maiti, "Unsteady Hydromagnetic Couette Flow in a Rotating System," Inter- 
national Journal of Engineering Science, Vol. 20, No. 9, 1982, pp. 989-999. doi:10.1016/0020-7225(82)90034-9

[3] R. Ganapathy, "A Note on Oscillatory Couette Flow in a Rotating System," Journal of Applied Mechanics, Vol. 61, No. 1, 1994, pp. 208-209. doi:10.1115/1.2901403

[4] A. S. Gupta, "Ekman Layer on a Porous Plate," Physics of Fluids, Vol. 15, No. 5, 1972, pp. 930-931. doi:10.1063/1.1694002

[5] B. S. Mazumder, "An Exact Solution of Oscillatory Couette Flow in a Rotating System," Journal of Applied Mechanics, Vol. 56, No. 4, 1991, pp. 1104-1107. doi:10.1115/1.2897694

[6] V. Vidyanidhi and S. D. Nigam, "Couette Flow between Rotating Parallel Plates under Constant Pressure Gradient," Journal of Mathematical Physics, Vol. 1, 1967, pp. 85 .

[7] R. N. Jana and N. Dutta, "Couette Flow and Heat Transfer in a Rotating System," Acta Mechanica, Vol. 26, No. 1-4, 1977, pp. 301-306. doi:10.1007/BF01177152

[8] K. D. Singh and R. Sharma, "Three Dimensional Couette Flow through Porous Media," Indian Journal of Pure and Applied Mathematics, Vol. 32, No. 12, 2001, pp. 18191829.

[9] K. D. Singh, M. G. Gorla and H. Rajhans, "A Periodic Solution of Oscillatory Couette Flow through a Porous Medium in Rotating System," Indian Journal of Pure and Applied Mathematics, Vol. 36, No. 3, 2005, pp. 151-159.

[10] M. Guria, R. N. Jana and S. K. Ghosh, "Unsteady Couette Flow in a Rotating System," International Journal of
Non-Linear Mechanics, Vol. 41, No. 6-7, 2006, pp. 838843. doi:10.1016/j.ijnonlinmec.2006.04.010

[11] B. K. Das, M. Guria and R. N. Jana, "Unsteady Couette Flow in a Rotating System," Meccanica, Vol. 43, No. 5, 2008, pp. 517-521. doi:10.1007/s11012-008-9130-x

[12] S. Das, S. L. Maji, M. Guria and R. N. Jana, "Unsteady MHD Couette Flow in a Rotating System," Mathematical and Computer Modelling, Vol. 50, No. 7-8, 2009, pp. 1211-1217.doi:10.1016/j.mcm.2009.05.036

[13] H. A. Attia, "Effect of Porosity on Unsteady Couette Flow with Heat Transfer in the Presence of Uniform Suction and Injection," Kragujevac Journal of Science, Vol. 31, No. 1, 2009, pp. 11-16.

[14] C. Israel-Cookey, E. Amos and C. Nwaigwe, "MHD Oscillatory Couette Flow of a Radiating Viscous Fluid in a Porous Medium with Periodic Wall Temperature," American Journal of Scientific and Industrial Research, Vol. 1, No. 2, 2010, pp. 326-331.

[15] B. G. Prasad and R. Kumar, "Unsteady Hydromagnetic Couette Flow through a Porous Medium in a Rotating System," Theoretical and Applied Mechanics Letters, Vol. 1, No. 4, 2011, Article ID: 042005. doi:10.1063/2.1104205

[16] S. Das, M. Jana and R. N. Jana, "Couette Flow through Porous Medium in a Rotating System," International Journal of Mathematical Archive, Vol. 2, No. 11, 2011, pp. 2318-2326.

[17] H. S. Carslaw and J. C. Jaeger, "Conduction of Heat in Solids," Oxford University Press, Oxford, 1959, p. 297. 